\title{
Datasus: possibilidade de contribuição no combate à violência contra a mulher no Rio De Janeiro
}

\author{
Datasus: possibility of contribution in the fight against violence against women in Rio De Janeiro \\ Datasus: posibilidad de contribución en la lucha contra la violencia contra las mujeres en Rio De Janeiro
}

\begin{abstract}
RESUMO
Objetivo: analisar a comparação entre os dados disponíveis no DATASUS e Instituto de Segurança Pública do Estado do Rio de Janeiro referentes à violência contra a mulher. Método: Trata-se de um estudo descritivo e retrospectivo, desenvolvido a partir dos dados disponíveis e consultados nas bases do Departamento de Informática do Sistema Único de Saúde (DATASUS) e Instituto de Segurança pública do Rio de Janeiro (ISP). Resultados: Número de notificações e ocorrências dos anos de 2014 a 2018, dos dados apresentados pelo DATASUS e Instituto de segurança pública do Rio de Janeiro. Conclusão: O Departamento de Informática do Sistema Único de Saúde pode contribuir para o desenvolvimento de novas políticas no enfrentamento à violência contra a mulher pois seus dados fornecem as informações para a criação de projetos, leis e métodos de educação em saúde.
\end{abstract}

DESCRITORES: Violência contra a mulher; DATASUS; Políticas de saúde.

\section{ABSTRACT}

Objective: Analyze the comparison between the data available at DATASUS and the Rio de Janeiro State Public Security Institute regarding violence against women. Method: This is a descriptive and retrospective study, developed from the available data and consulted in the databases of the Department of Informatics of the Unified Health System (DATASUS) and the Institute of Public Security of Rio de Janeiro (ISP). Results: Number of notifications and occurrences from the years 2014 to 2018, of the data provided by DATASUS and the Rio de Janeiro Institute of Public Security.Conclusion: Department of Informatics of the Unified Health Systemcan contribute to the development of new policies to combat violence against women, as its data provide information for the creation of health education projects, laws and methods.

DESCRIPTORS: Violence against women; DATASUS; Health policies.

\section{RESUMEN}

Objetivo: Analizar la comparación entre los datos disponibles en DATASUS y en el Instituto de Seguridad Pública del Estado de Río de Janeiro sobre violencia contra las mujeres. Método: Se trata de un estudio descriptivo y retrospectivo, desarrollado a partir de los datos disponibles y consultados en las bases de datos del Departamento de Informática del Sistema Único de Salud (DATASUS) y el Instituto de Seguridad Pública de Río de Janeiro (ISP). Resultados: Número de notificaciones y ocurrencias de los años 2014 a 2018, de los datos proporcionados por DATASUS y el Instituto de Seguridad Pública de Río de Janeiro. Conclusión: El Departamento de Informática del Sistema Único de Salud puede contribuir al desarrollo de nuevas políticas para enfrentar la violencia contra las mujeres, ya que susdatos brindan información para la creación de proyectos, leyes y métodos de educación en salud.

DESCRIPTORES: Violencia contra la mujer; DATASUS; Políticas de salud.

RECEBIDO EM: 09/10/2020 APROVADO EM: 21/10/2020

\section{Ísis da Costa Bezerra}

Graduanda em Enfermagem pela Faculdade Adventista da Bahia. BA-Brasil.

ORCID: 0000-0003-2482-7136

\section{Rebeca Cardoso da Rocha}

Graduanda em Enfermagem pela Faculdade Adventista da Bahia. BA-Brasil.

ORCID: 0000-0003-3726-4598 


\section{Gabriel Lucas Pereira Guimarães}

Tecnólogo em Secretariado, Pós-graduando em Administração e Auditoria em Serviços de Saúde, Graduando em Enfermagem pela Faculdade Adventista da Bahia. BA-Brasil.

ORCID: 0000-0002-4669-6715

\section{Samylly dos Santos Santana}

Graduanda em Enfermagem pela Faculdade Adventista da Bahia. BA-Brasil.

ORCID: 0000-0001-5689-3723

\section{Quéren Gabriele Cunha Silva}

Graduanda em Enfermagem pela Faculdade Adventista da Bahia. BA-Brasil.

ORCID: 0000-0002-4489-7994

\section{Paula Paulina Costa Tavares}

Enfermeira. Mestrado Profissional em Promoção da saúde pelo Centro universitário Adventista de São Paulo. Docente do curso de Enfermagem da Faculdade Adventista da Bahia. BA-Brasil.

ORCID: 0000-0002-9275-8884

\section{INTRODUÇÃO}

A violência contra a mulher é definida pelas Nações Unidas como "qualquer ato de violência de gênero que resulte ou possa resultar em danos ou sofrimentos físicos, sexuais ou mentais para as mulheres, inclusive ameaças de tais atos, coerção ou privação arbitrária de liberdade, seja em vida pública ou privada". Configura como violação dos direitos humanos e problema de saúde pública, das quais destaca-se a violência sexual cometida por parceiros ${ }^{(1)}$. A violência contra a mulher se apresenta como uma forma de legitimação de poder do homem sobre a mulher, sendo por isso denominada de violência de gênero, produto da organização social, alicerçado nas desigualdades entre os sexos, estabelecendo-se como um campo de luta estruturada pelas diferenças de poder entre homens e mulheres ${ }^{(2)}$.

No Brasil estima-se que a cada ano, 1,3 milhões de mulheres sofrem algum tipo de violência ${ }^{(3)}$. A partir da comparabilidade entre grupos de 100 mil mulheres constatou-se que entre os anos de 2007 a 2017 houve crescimento de $20,7 \%$ da taxa de homicídios contra mulheres, essa taxa passou de 3,9\% para 4,7\% de mulheres assassinadas por grupo de 100 mil. Entre os anos de 2012 a 2017 foi observado crescimento de $1,7 \%$, seguido de $5,4 \%$ no último ano $^{(4)}$. O estado do Rio de janeiro destaca-se no que se refere a apresentação
A partir da comparabilidade entre grupos de 100 mil mulheres constatou-se que entre os anos de 2007 a 2017 houve crescimento de $20,7 \%$ da taxa de homicídios contra mulheres, essa taxa passou de 3,9\% para $4,7 \%$ de mulheres assassinadas por grupo de $100 \mathrm{mil}$. de dados, dando visibilidade e transparência à questão da violência contra a mulher, sendo pioneiro no Brasil no desenvolvimento sistemático e divulgação de estatísticas por meio de dossiê tendo como base os registros de ocorrências das delegacias de polícia civil do estado, o que possibilita uma avaliação, comparação e acompanhamento dos indicadores de violências sofridas por mulheres ${ }^{(5)}$.

Em situações de violência contra mulher, preconiza-se o direcionamento ou orientação da vítima a comparecer à delegacia de mulheres para registrar boletim (registro) de ocorrência. Em caso de mulheres em situação de violência, que não sejam idosas ou deficientes, as equipes de saúde devem informar sobre os serviços da rede de proteção social e sobre a importância da denúncia, mas não devem comunicar ou denunciar o caso sem a sua autorização. Em todos os casos, o atendimento deve respeitar a autonomia da mulher e seu direito de escolha, obedecendo às normativas do Ministério da Saúde ${ }^{(6)}$. Compete ao profissional de saúde realizar a notificação compulsória em caso de violência contra a mulher, o que fornece dados para o Departamento de Informática do Sistema Único de Saúde (DATASUS), também orientar essas mulheres como proceder. A notificação compulsória de violências interpessoais e autoprovocadas no âmbito da saúde não configura como denúncia, mas sim como um instrumento 
de garantia de direitos, prerrogativa muitas vezes esquecida ${ }^{(6)}$.

Haja vista que a utilização e aplicação de dados do departamento de informática do SUS pode possibilitar a otimização de tempo, melhoria no atendimento e registro de casos, esta pesquisa tem por objetivo analisar a comparação entre os dados disponíveis no DATASUS e Instituto de Segurança Pública do Estado do Rio de Janeiro referentes à violência contra a mulher.

\section{METODOLOGIA}

Trata-se de um estudo descritivo e retrospectivo, desenvolvido a partir dos dados disponíveis e consultados nas bases do Departamento de Informática do Sistema Único de Saúde (DATASUS) e Instituto de Segurança pública do Rio de Janeiro (ISP) referente aos anos de 2014 a 2018.

A busca ocorreu no período de março a agosto de 2020, sendo possível levantar o número de notificações compulsórias e registros de ocorrências no estado do Rio de Janeiro. Os dados das bases foram processados e sumarizados em tabela.

\section{RESULTADOS E DISCUSSÃO}

A Tabela 1 mostra o quantitativo referente ao número de notificações e ocorrências dos anos de 2014 a 2018 dos dados apresentados pelo DATASUS e ISP do Rio de Janeiro, possibilitando um comparativo entre as bases. Ao analisar a tabela, é possível perceber que as notificações

variaram entre $9,1 \%$ e $13,4 \%$ do número de ocorrências nos referidos anos. Com relação ao número total de ambos os registros, sem considerar o ano de 2018, devido ausência de informação do número de notificações, o percentual corresponde a $11,1 \%$, ou seja, muito abaixo do número de ocorrências, o que aponta importante disparidade e remete, no mínimo, à reflexão sobre as causas dessa comparação desproporcional.

\section{Datasus: discrepâncias com secreta- ria de segurança pública}

O DATASUS surgiu em 1991 com a criação da Fundação Nacional de Saúde (Funasa) com a finalidade de trazer dados quantitativos a respeito dos agravos notificados pelas unidades de saúde em todos os níveis da rede, proporcionando acesso a quem deseja conhecer essas informações além de fornecer dados para que os gestores possam desenvolver estratégias objetivando a melhoria na prestação dos serviços de saúde ${ }^{(9)}$. O sistema também pode disponibilizar informações que sirvam para subsidiar análises objetivas da situação sanitária, tomadas de decisões baseadas em evidências e elaboração de programas de ações de saúde, possibilidades estas, prejudicadas pela não conformidade entre os registros legais e os dados do sistema ${ }^{(7)}$.

Teoricamente os números de notificações do DATASUS deveriam ser iguais ou próximos aos números de casos de denúncias junto às secretarias de segurança

\section{Tabela 1: Notificações e ocorrências.}

\section{DATASUS}

$\begin{array}{cccc}\text { Ano de notificação } & \begin{array}{c}\text { Número de notifi- } \\ \text { cações }\end{array} & \begin{array}{c}\text { Ano de ocorrências } \\ \text { (casos) }\end{array} & \begin{array}{c}\text { Número de Ocor- } \\ \text { rências }\end{array} \\ 2014 & 22.835 & 2014 & 169.431 \\ 2015 & 14.730 & 2015 & 147.407 \\ 2016 & 12.168 & 2016 & 132.607 \\ 2017 & 12.672 & 2017 & 111.877 \\ 2018 & ----- & 2018 & 121.077 \\ \text { Total } & 62.405 & \text { Total } & 682.399\end{array}$

pública, contudo, é possível identificar discrepâncias entre os números notificados e as ocorrências, o que por sua vez prejudica a finalidade da base de dados do Ministério da Saúde. Faz-se necessário que haja forte metodologia para o combate à subnotificação desse sistema, permitindo o acompanhamento dos avanços e dificuldades na implementação de políticas públicas e condições de saúde da população, contribuindo também para o fortalecimento do Sistema Único de Saúde (SUS) (7). A interação e o relacionamento entre os serviços de proteção podem garantir eficácia na realização do atendimento nos casos de violência contra a mulher, o que torna necessária a comunicação entre todos os serviços disponíveis para atender a vítima, possibilitando maior proteção e prevenção de danos ${ }^{(10)}$.

Devido as disparidades encontradas nas referidas bases de dados, é possível entender que a subnotificação tem fator influenciador na implementação de políticas de saúde pública ${ }^{(11)}$. Tal fato pode ser observado ao analisar o comparativo entre notificações e ocorrências, onde o DATASUS além de não dispor de todos os dados, também não contempla todos os anos em sua plataforma, e vários podem ser os motivos dessa desconformidade, dentre eles, a subnotificação. O ISP apesar de disponibilizar números referentes apenas aos anos entre 2014 e 2018, traz um quantitativo mais realista que pode contribuir para geração de políticas na esfera da seguranç ${ }^{(7,8)}$.

A Secretaria Especial de Políticas para as Mulheres determina objetivos com a finalidade de enfrentamento à violência contra a mulher, sendo alguns deles, a garantia de atendimento integral a essa mulher, definição de normas do atendimento que deve ser humanizado, e traz também a integração de redes locais, regionais e nacionais, envolvendo múltiplos órgãos como polícia, bombeiros e serviços de saúde, dentre outros ${ }^{(12)}$.

No estado do Rio de Janeiro a Subsecretaria de Políticas para as Mulheres SSPM é responsável pela elaboração de políticas públicas para esse público, tendo 
dentre suas atribuições "Articular com órgãos das três esferas de governo e entidades da sociedade civil, com o objetivo de assegurar a implementação dos Planos de Políticas para as Mulheres," atuando em conjunto com a Superintendência de Enfrentamento à Violência contra a Mulher (SUPEV) para desenvolvimento e implementação de políticas para as mulheres ${ }^{(13)}$.

$\mathrm{Na}$ área da violência contra a mulher são necessárias políticas que assegurem a capacitação dos agentes envolvidos, principalmente nas questões de gênero, com objetivo de melhor compreensão do fenômeno e assistência adequada às vítimas. São essenciais estratégias que possibilitem denúncia, proteção e apoio à mulher, de forma articulada entre as esferas de atendimento como saúde, educação, assistência social, segurança pública, entre outros, resultando em melhor enfrentamento, prevenção, combate, assistência e garantia de direitos ${ }^{(14)}$.

As estratégias de registro de informações, por mais que careçam de aprimoramentos, pretendem contribuir para gerar dados que dimensionam a magnitude da violência contra mulheres como questão a ser enfrentada por diferentes setores e categorias profissionais. Contudo, elas não são canais exclusivos de visibilidade desse fenômeno, pesquisas qualitativas com diferentes enfoques, também contribuem para sua desnaturalização, além de atestar sua relevância para se pensar estratégias de enfrentamento da violência contra mulher. "Essa "invisibilidade" da violência se insinua nos serviços de saúde, que se restringem, na maioria das vezes, a tratar os efeitos das violências vivenciadas por mulheres" (11).

\section{O Profissional De Saúde E A Notificação}

$\mathrm{O}$ enfrentamento à violência contra as mulheres não deve se restringir às ações nas áreas da segurança e assistência social, deve sim, envolver diferentes setores do Estado. Os Planos Nacionais de Políticas para Mulheres, defendem a articulação entre um eixo vertical, que estabelece políticas e serviços entre as esferas de governo de modo a otimizar os recursos existentes e potencializar os resultados, com um eixo

horizontal, por meio do qual os serviços devem fazer parte de uma rede intersetorial que possibilite $\mathrm{o}$ atendimento integral às mulheres ${ }^{(15)}$.

As estratégias

de registro de

informações, por

mais que careçam

de aprimoramentos,

pretendem contribuir

para gerar dados

que dimensionam

a magnitude da

violência contra

\section{mulheres como}

questão a ser

enfrentada por

diferentes setores

e categorias

profissionais.

Geralmente o primeiro serviço procurado pela mulher vítima de violência é a Rede de Atendimento à Saúde (RAS), em seus diversos níveis, a qual compõe a rede intersetorial. Cabe à equipe de saúde identificar e notificar os casos violência, podendo configurar como omissão o não cumprimento da notificação. Torna-se evidente a necessidade do compromisso dos profissionais de saúde perante às pacientes, apesar de não ser o setor responsável por queixas crimes, a notificação contribui para o combate à vio- lência contra a mulher ${ }^{(16)}$. Quando se trata do preenchimento da ficha de notificação, a Lei Federal n ${ }^{\circ} 10.788$, estabelece, em seu artigo $5^{\circ}$ que: "A inobservância nesta Lei constitui infração da legislação referente à saúde pública, sem prejuízo das sanções penais cabíveis" ${ }^{(17)}$.

Entre as etapas de acolhimento, atendimento e notificação, deve-se proceder às normas na rede de proteção social. A ficha de notificação é um instrumento disparador da linha de cuidado às pessoas em situação de violência e de extrema ajuda a quem precisa ${ }^{(6)}$. A notificação compulsória deve ser do conhecimento geral e é obrigatória a todos os profissionais de saúde: médicos, enfermeiros, odontólogos, médicos veterinários, biólogos, biomédicos, farmacêuticos e outros no exercício da profissão, bem como os responsáveis por organizações e estabelecimentos públicos e particulares de saúde e de ensino, em conformidade com os arts. $7^{\circ}$ e $8^{\circ}$, da Lei $\mathrm{n}^{\circ} 6.259$, de 30 de outubro de $1975^{(18)}$.

Ao profissional de saúde compete evitar futuros agravos às vítimas decorrentes de violências, tal ação torna-se possível por meio de detecção precoce de situações de violência. $\mathrm{O}$ profissional deve fazer uso de métodos multifatoriais (acolhimento, empatia, vínculo de confiança etc.) e multiprofissionais que possibilitem uma melhor assistência, orientação e prevenção, evitando danos maiores para a vítima no futuro. A adequada abordagem do profissional possibilitará à mulher uma percepção e embasamento no assunto ${ }^{(19)}$. A equipe multidisciplinar deve atuar de maneira conjunta evitando descontinuidade na prestação da assistência às vítimas e ao processo de notificação, esta atuação deve se dar em todas as etapas desde o combate até o cuidado ${ }^{(16)}$. A equipe de saúde deve então agir de maneira multifacetada promovendo métodos diversos que visem reeducação e proteção, e não só a notificação ${ }^{(20)}$.

\section{CONCLUSÃO}

Ao analisar os dados referentes à violência contra mulher disponíveis nos bancos de dados do DATASUS e ISP, foi possível iden- 
tificar grande disparidade entre o número de notificações e ocorrências, o que aponta falhas na rede de atenção à saúde demonstradas pela subnotificação dos casos desse tipo de violência. O DATASUS pode contribuir para o desenvolvimento de novas políticas no enfrentamento à violência contra a mulher pois seus dados fornecem informações para a criação de projetos, leis e métodos de educação em saúde. Contudo a discrepância em relação a outras bases de dados é um fator que pode causar uma micro visão do cenário em que o Estado do Rio de Janeiro se encontra, o que, ocasionalmente, não expressa a realidade como um todo e pode gerar políticas deficitárias. Melhorias com intuito de evitar subnotificações são necessárias para contemplar de maneira adequada a prestação dos serviços e ações em saúde.

É necessário haver planejamento, colaboração e articulação entre as Secretarias de Segurança Pública e de Saúde, na figura das unidades responsáveis pelas notificações, para que assim seja possível o surgimento de novas leis e políticas de combate a violência contra a mulher, assim como também reforçar leis já vigentes.

\section{REFERÊNCIAS}

1. Folha informativa- violência contra a mulher [internet]. Banco de notícias; 2017 [cited 2020 May 13]. Disponivel em: https:// www.paho.org/bra/index.php?option=com_content\&view=article\&id=5669:folha-informativa-violencia-contra-as-mulheres\&ltemid $=820$

2. Heise LL, Pitanguy J, Germain A, compilers. Violence against women: the hidden health burden [bibliography]. Washington, D.C.: The World Bank; 1994. 86 p.

3.Instituto de Pesquisa Econômica Aplicada - Ipea. Índice de violência doméstica é maior para mulheres economicamente ativas: Estudo inédito do Ipea baseado em dados da Pnad apresenta possíveis explicações para o fenômeno [Internet]. Brasília; 2019 [cited 2020 Aug 7]. Disponivel em:https://www.ipea.gov.br/portal/index.php?option=com_content\&view=article\&id=34977

4.Cerqueira D, Lima RS, Bueno S, Neme C, Ferreira H, Alves PP, et al. Atlas da Violência [Internet]. Fórum Brasileiro de Segurança Pública; 2019 [cited 2020 Aug 7]. Disponível em: https://www. ipea.gov.br/portal/images/stories/PDFs/relatorio_institucional/180604_atlas_da_violencia_2018.pdf

5.Instituto de Segurança Pública ISP. Dossiê Mulher 2019 [Internet]. Rio de Janeiro; 2020 [cited 2020 May 5]. Disponível em: http://arquivos.proderj.rj.gov.br/isp_imagens/uploads/DossieMulher2019.pdf

6. Orientações para notificação e atendimento, 2018. Ministério da saúde do Brasil [Internet]. 2018 [cited 2020 May 13]; Disponivel em: https://www.saude.gov.br/vigilancia-em-saude/ vigilancia-de-violencias-e-acidentes-viva/vigilancia-de-violencias/orientacoes-para-notificacao-e-atendimento

7.Ministério da saúde datasus [internet]. 2020. tabnet: informações de saúde; [revised 2020 May 13; cited 2020 May 13]; Disponivel em: https://datasus.saude.gov.br/informacoes-de-saude-tabnet/.

8. Instituto de Segurança Pública ISP. isp dados visualização [Internet]. Rio de Janeiro; 2020 [cited 2020 May 5]. Disponível em: http://www.isp.rj.gov.br/

9. Brasil. Decreto n 100, de 16 de Abril 1991 e ratificado em 19 de Abril 1991 - Publicação Original [Internet]. Brasília; 1991 [cited 2020 May 9]. Disponível em: https://www2.camara.leg.br/legin/ fed/decret/1991/decreto-100-16-abril-1991-342868-publicacaooriginal-1-pe.html

10.Machado DF, Almeida MAS, Dias A, Bernardes JM, Castan- heira ERL. Violência contra a mulher: o que acontece quando a Delegacia de Defesa da Mulher está fechada? Ciênc. saúde coletiva. 2020;25(2)

11. Kind L., Orsini M., Nepomuceno V., Gonçalves L., Souza G., Subnotificação e (in)visibilidade da violência contra mulheres na atenção primária à saúde. Cad. Saúde Pública. 2013; 29(9):18051815.

12. II Plano Nacional de Políticas para as Mulheres: Secretaria Especial de Políticas para as Mulheres [Internet]. Brasília; 2005 [cited 2020 Aug 25]. Disponível em: www.presidencia.gov.br/ spmulheres

13. Secretaria de Desenvolvimento Social e Direitos Humanos: Subsec Políticas para Mulheres [Internet]. Rio de Janeiro; 2020 [cited 2020 Aug 20]. Disponivel em: http://www.rj.gov.br/secretaria/PaginaDetalhe.aspx?id_pagina=3491

14. Cavalcanti ECT, Oliveira RC. Políticas públicas de combate à violência de gênero: a rede de enfrentamento à violência contra as mulheres. Rev. de Pesquisa Interdisciplinar. 2017;2(2):192206.

15. Passinato W, Santos C. Mapeamento das Delegacias da Mulher no Brasil [Internet]. Campinas; 2008 [cited 2020 Aug 25]. Disponivel em: https://www12.senado.leg.br/institucional/omv/entenda-a-violencia/pdfs/mapeamento-das-delegacias-da-mulher-no-brasil

16. Hasse M, Vieira EM. Como os profissionais de saúde atendem mulheres em situação de violência? Uma análise triangulada de dados. Saúde em Debate. 2014;38(102):482-493.

17. Brasil. Lei No 10.778, de 24 de Novembro De 2003. [Internet]. Brasília; 2003 Nov 24 [cited 2020 Jul 27]. Disponível em: http://www.planalto.gov.br/ccivil_03/LEIS/2003/L10.778.htm

18. portaria $n^{\circ} 104$, de 25 de janeiro de 2011. Ministério da saúde do brasil [Internet]. 2018 [cited 2020 May 13]; Disponível em: http://bvsms.saude.gov.br/bvs/saudelegis/gm/2011/ prt0104_25_01_2011.html

19. Aguiar RS. O cuidado de enfermagem à mulher vítima de violência doméstica. R. Enferm. Cent. O. Min. 2013;3(2):723-731.

20. Freitas RJM, Sousa VB, Costa TSC, Feitosa TMM, Monteiro ARM, Moura NA. Atuação dos enfermeiros na identificação e notificação dos casos de violência contra a mulher. HU Revista. 2017;43(2):91-9 